\title{
Fat-poor renal angiomyolipoma combined with pseudoaneurysm: a case report
}

\author{
Jing Chong ${ }^{1}$, Junpeng Zhang ${ }^{2}$, Chunping Ning ${ }^{1}$, Liang Zhang ${ }^{1}$, Wei Zhao ${ }^{1}$, Yongmei Sun ${ }^{1}$ \\ ${ }^{1}$ Department of Abdominal Ultrasound, the Affiliated Hospital of Qingdao University, Qingdao, China; ${ }^{2}$ Department of Abdominal Ultrasound, \\ Shandong Provincial Hospital Affiliated to Shandong University, Jinan, China \\ Correspondence to: Yongmei Sun. Department of Abdominal Ultrasound, the Affiliated Hospital of Qingdao University, Qingdao 266000, China. \\ Email: symdoc@163.com.
}

\begin{abstract}
Renal angiomyolipoma (RAML), a rare benign mesenchymal tumor, consists of variable proportions of thick-walled blood vessels admixed with adipose tissue and bundles of smooth muscle. Rarely, RAML angiomyolipoma may be associated with pseudoaneurysm, spontaneous rupture and hemorrhage. we herein present an exceptional case of fat-poor RAML containing a large pseudoaneurysm in a 48-yearold woman with known pulmonary lymphangioleiomyomatosis (PLAM), who was admitted to hospital due to right flank pain for about two months. To avoid risk of rupture of the pseudoaneurysms, transcatheter arterial embolization (TAE) procedure was performed, at the same time taking into account of the possibility of malignancy in such a large neoplasm, right radical nephrectomy was taken five days later. This case was benign and the 1-year follow-up ultrasonic examination showed no recurrence. This is the first report that the patient had a sporadic giant RAML with pseudoaneurysm which was not associated with the TSC, and to our knowledge, such cases have not been explored in literature. This case reminds us that giant sporadic RAML with pseudoaneurysm, although rare, does exist, and we should improve the understanding of the neoplasm. Early detection and therapeutic interventions of pseudoaneurysm in an AML patient can reduce serious complications.
\end{abstract}

Koywords: Renal angiomyolipoma (RAML); pseudoaneurysm; ultrasonography; pulmonary lymphangioleiomyomatosis (PLAM); case report

Submitted Feb 24, 2020. Accepted for publication May 12, 2020.

doi: 10.21037/apm-20-475

View this article at: http://dx.doi.org/10.21037/apm-20-475

\section{Introduction}

Renal angiomyolipoma (RAML) is the most common benign neoplasms of the kidney with an incidence of $0.3-3 \%(1,2)$, even more infrequent during pregnancy (3). It either occurs sporadically or as multiple lesions associated with tuberous sclerosis complex (TSC), an autosomal dominant phacomatosis involving multiple organs and multiple systems (4). Pseudoaneurysm formation is usually noted in TSC-associated RAMLs and is extremely rare in the sporadic variety. Arterial pseudoaneurysm can cause free rupture and potentially life-threatening hemorrhage which has to be timely treated. Pulmonary lymphangioleiomyomatosis (PLAM) is a hamartomatous disorder that occurs more common in women of reproductive years, and shares the same morphologic and immunohistochemical characteristics with RAMLs. Our case was unique in that this patient had a sporadic giant RAML with pseudoaneurysm which was not associated with the TSC. We present the following case in accordance with the CARE reporting checklist.

\section{Case presentation}

A 48-year-old woman with history of PLAM was admitted to the Department of Urology complaining of right flank pain for about two months. Nine years ago, she was 

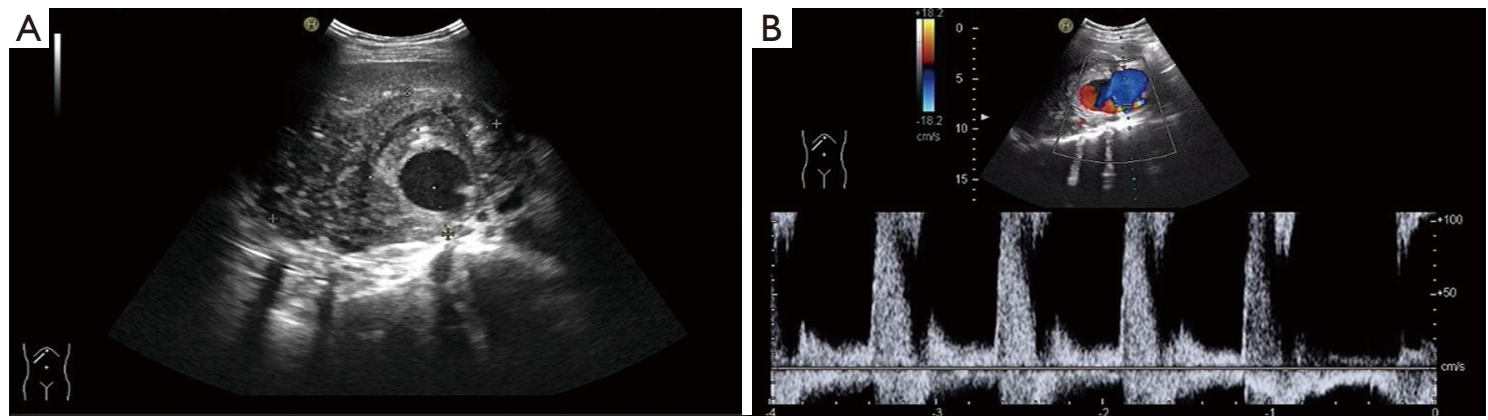

Figure 1 Ultrasonic appearance of the renal mass. (A) A large heterogeneous hypoechoic mass, containing an anechoic area was detected in the right kidney; (B) pulsed Doppler sonogram showed a typical pattern known as the to-and-fro sign in the anechoic intratumoral neoplasm.
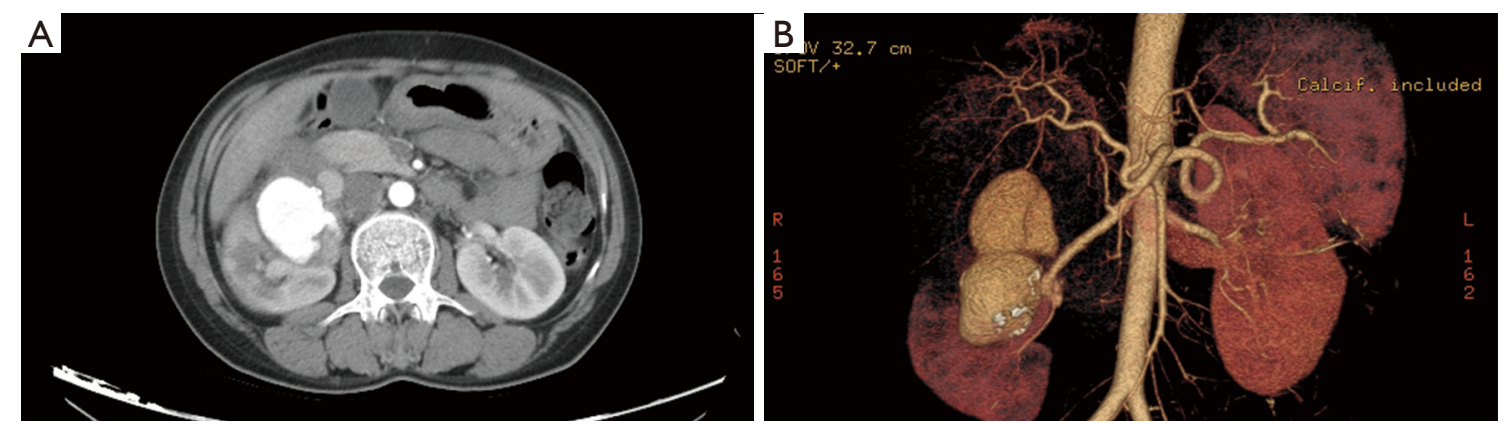

Figure 2 Axial on contrast enhanced CT showed moderate heterogenous enhancement of the right renal mass, with a well-defined area with enhancement similar to arterial enhancement in the center of the renal mass (A); 3D visualization of pseudoaneurysm projecting from the right renal artery $(\mathrm{B})$.

diagnosed as PLAM by lung biopsy because of spontaneous pneumothorax. No positive family history. Physical examination revealed a palpated mass in the right kidney. Laboratory tested were showed as follows: serum creatinine, $45 \mu \mathrm{mol} / \mathrm{L}$ (normal range, $31-133 \mu \mathrm{mol} / \mathrm{L}$ ); urea nitrogen, $2.3 \mathrm{mmol} / \mathrm{L}$ (normal range, $2.1-7.1 \mathrm{mmol} / \mathrm{L}$ ); urine occult blood, 2+ (normal, -). Complete blood count and hepatic function were normal, and all tumor markers AFP, CA19-9 and CEA were negative.

Sonographic examination of the abdomen revealed an anechoic mass measuring approximately $7.3 \mathrm{~cm} \times 3.8 \mathrm{~cm} \times$ $5.2 \mathrm{~cm}$ in a heterogeneous mass measuring size $11.5 \mathrm{~cm} \times$ $7.4 \mathrm{~cm} \times 6.8 \mathrm{~cm}$ in the upper and middle pole of the right kidney (Figure 1A). "Swirling" blood flow were found in the anechoic mass by Color Doppler and Pulsed Doppler, which can detect the arterial blood flow spectrum, PSV: $132 \mathrm{~cm} / \mathrm{s}$, RI: 0.73 (Figure 1B). By sonographic examination, she was diagnosed as renal mass complicated with intratumor pseudoaneurysm.
Abdominal computed tomography (CT) scan with contrast demonstrated a large exophytic heterogeneous mass, $12.3 \mathrm{~cm} \times 10.9 \mathrm{~cm} \times 8.3 \mathrm{~cm}$ in size, being in upper and middle pole of right kidney displacing and compressing residual renal parenchyma. There was evidence of a large quasi-circular vascular sac like lesion measuring $8.2 \mathrm{~cm} \times$ $4.5 \mathrm{~cm} \times 3.6 \mathrm{~cm}$ in the center of the heterogeneous lesion which was enhanced in arterial phase and persistent filling during portal venous phase (Figure $2 A$ ). Three-dimensional volume rendered computed tomography angiography (CTA) shows a saccular pseudoaneurysm of the right renal artery (Figure 2B). CT of the chest was performed and found multiple small thin-walled cysts scattered in the central and peripheral lung field (Figure 3), compatible with the diagnosis of PLAM.

To avoid a significant risk of hemorrhage, transcatheter arterial embolization (TAE) procedure was recommended and accepted, and intraoperative findings were consistent with pseudoaneurysm. Five days later, the patient was taken 


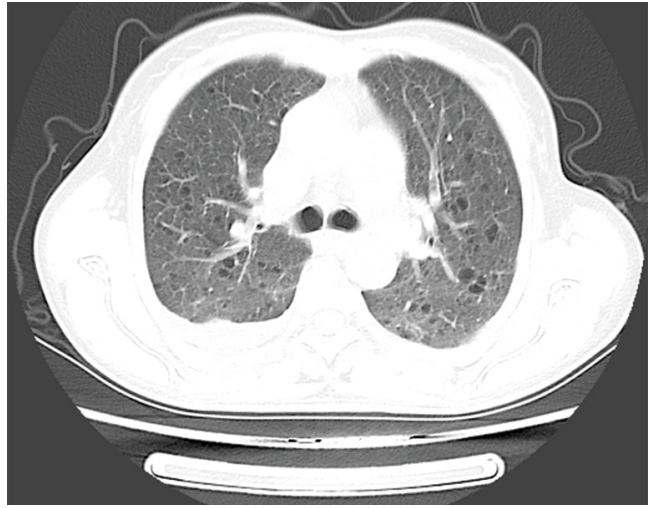

Figure 3 High-resolution CT of the chest shows well defined thin-walled cysts randomly scattered throughout both lungs.

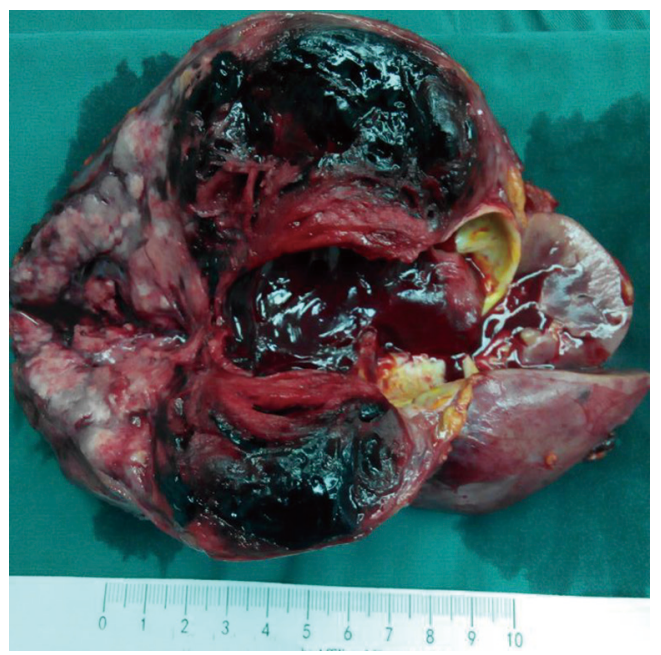

Figure 4 The surgically resected specimen showed a large tumor grew extranephridially with large areas of necrosis and hemorrhage within the tumour. for surgery and a total nephrectomy was performed, in line with the surgical indications. The right kidney was shown in Figure 4. A large complex mass located in the middle and upper part of the right kidney occupying $80 \%$ of the renal volume. Histological examination revealed that the major components of the neoplasm were smooth muscle cells, blood vessels, with only small amounts of mature adipose cells (Figure 5A). Immunohistochemically, smooth muscle cells stained positively for HMB-45 (Figure 5B), SMA, S-100 $(+)$ and $\mathrm{Ki}-67$. The Ki-67 labeling index was about $6 \%$. Accordingly, a final diagnosis of AML with large patchy necrosis and bleeding on right kidney was made. There were no diagnostic challenges. Nineteen days after the surgery, the patient was discharged. The patient recovered well, with no evidence of local recurrence or adverse event during postoperative follow-up period from January 27, 2019 to January 15,2020 . In addition, the patient's medical history was shown in timeline in Figure 6.

\section{Discussion}

Most cases of renal AMLs present as an isolated lesion while approximately $20 \%$ of patients have an association with the TSC (5). TSC-associated RAMLs are more likely to grow larger size, and have a greater risk of lifethreatening hemorrhage compared with isolated form. The patient in our reported case was finally diagnosed as AML occurred as sporadic entities, instead of an incomplete expression of TSC. RAMLs accompanied PLAM are rare disease of unknown etiology, which belongs to tumors with perivascular epithelioid cell tumor (PEComa). Both of the lesions can occur separately or simultaneously and such diseases can also be found in in patients with TSC at the
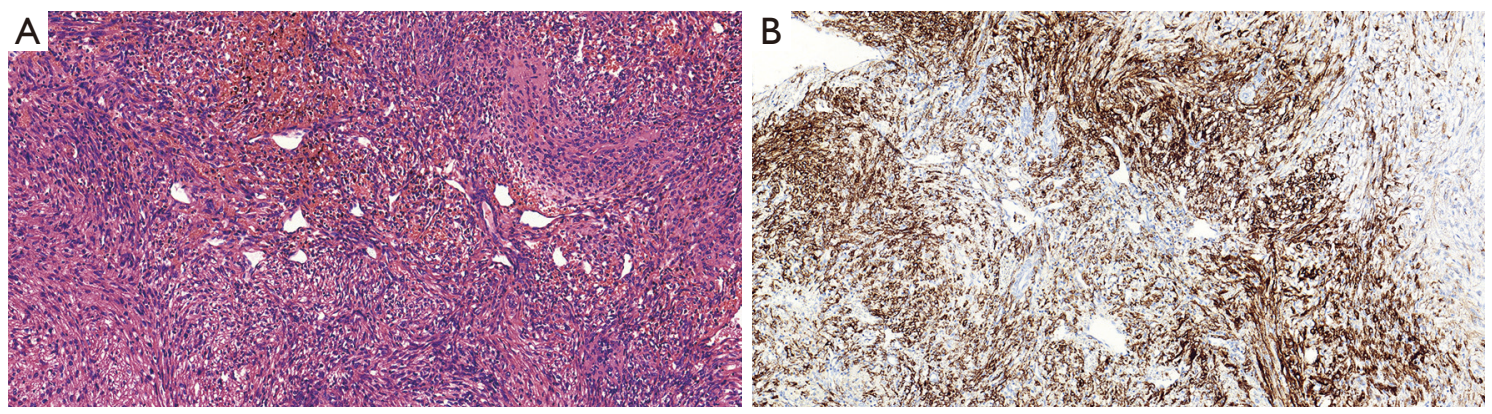

Figure 5 Histologic and immunohistochemical findings: (A) hematoxylin and eosin $(\times 200)$ stain showed tumor composed of smooth muscle, thick-walled blood vessels, with only small amounts of fat; (B) immunohistochemistry demonstrated positivity for HMB-45 ( $\times 200)$. 


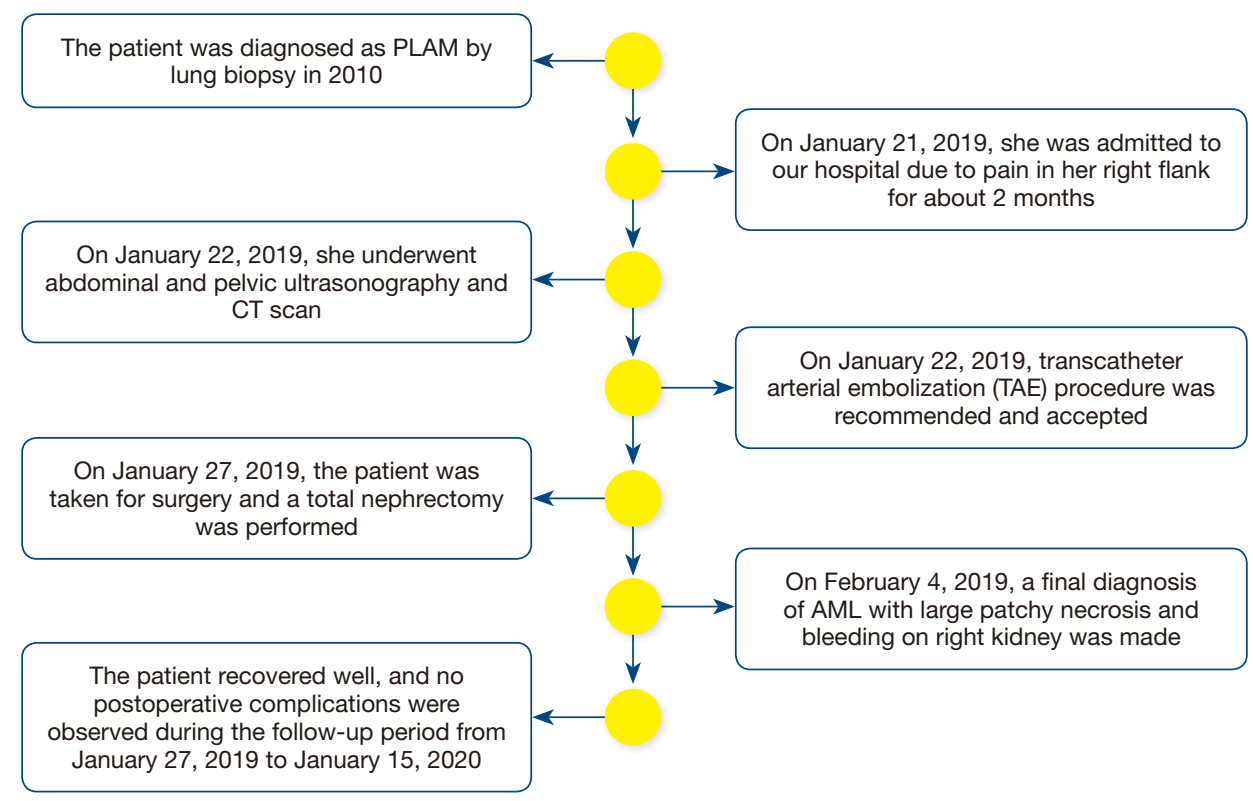

Figure 6 The timeline of the patient's medical historical.

same time. So, doctors should keep in mind the possibility of RAML in patients diagnosed with PLAM. Our case was striking because of large size of the tumor accompanied PLAM and complicated with large pseudoaneurysm.

Large RAML can cause serious complications, and pseudoaneurysm can lead to acute rupture hemorrhage, even hemorrhagic shock. It is clear that early detection and diagnosis of the disease can reduce the occurrence of serious complications. Although histological examination in combination with immunohistochemistry markers remains the gold standard for diagnosis, imaging studies are the main diagnostic tool to diagnose and differentiate between various causes of a renal mass, and assess the extent of hemorrhage. Imaging manifestations of AMLs depends on the proportions of fat, smooth muscle, vascular elements and on the presence of hemorrhage. Ultrasonically, AMLs typically appear as a circumscribed, highly reflective mass that is more echogenic than the central sinus fat. A fatty mass with areas of increased density on CT imaging remains the important diagnostic finding of renal AMLs. Unfortunately, AML may represent a diagnostic challenge for every imaging method in case of poor fat content, hemorrhage or necrosis, requiring percutaneous biopsy or surgery for diagnosis. In a study that in $5 \%$ of AML, there is insufficient fat to detect with imaging studies (6). In our case, as so little fat was discernible, and the tumor was adhered with surrounding tissue and accompanied with hemorrhage and necrosis, the imaging manifestations were so complex that it was difficult to distinguish AML from other renal masses. Whereas, Pulsed Doppler sonography showed characteristic to-and-fro flow within the mass as seen in our present patient and the diagnosis of pseudoaneurysms was established. To some extent, CTA may provide the similar information. However, CT imaging can't be performed in real-time and makes patients expose to ionizing radiation.

Most renal AMLs are asymptomatic and incidentally found when a patient receives renal sonography examination. In symptomatic patients, the classic symptoms of renal AMLs include hematuria, flank pain and mass palpation, even bleeding and rupture. Due to absence of elastic tissue of the vessels, and as the neoplasm grows, blood flow entering the neoplasm increases, leading to vessel dilatation and, predisposing to pseudoaneurysm formation or spontaneous hemorrhage. Intratumoral or perinephric hemorrhage is the usual complication of large AMLs, whereas pseudoaneurysm appears to be unusual. Only a few similar cases have been reported (7) and most of them are TSC-associated RAMLs. In general, lesions greater than $4 \mathrm{~cm}$, multicentric lesions and those associated with TSC have increased risk of hemorrhage (8). In the present case, the neoplasm was large, and in this setting, neoplastic angiorrhexis of the blood-supply artery led to the formation of pseudoaneurysm. 
As reported, some patients with AML present with severe complications and morbidity, such as hypovolemic shock following massive retroperitoneal bleeding, known as Wunderlich's syndrome (9), occurring in $10 \%$ of patients (10). Therefore, early detection and therapeutic interventions of pseudoaneurysm in an AML patient are of vital importance. Various management modalities available for AMLs primarily depend upon the size of the neoplasm, clinical presentation, risk stratification for hemorrhage and malignant potential. Classical surgical approach with a total or partial nephrectomy and selective TAE are usually alternative. In our case, taking into account of the possibility of malignancy in such a large renal neoplasm and a high risk of rupture of the pseudoaneurysms, active arterial embolization and right radical nephrectomy were taken.

The advantage of our case is that the imaging modalities (such as CT, ultrasound) and clinical data are very comprehensive, which is particularly essential for the retrospective study of the disease. However, our case still has certain limitations. In our case, there is insufficient fat to detect with imaging studies so that we did not make a correct diagnosis of RAML. There is no doubt that the final molecular pathology is still gold standard for diagnosis.

\section{Conclusions}

Giant sporadic RAML with pseudoaneurysm, although rare, does exist. In summary, this case demonstrates an uncommon case of minimal fat AML with pseudoaneurysm and highlights the possibility of AML in patients diagnosed with PLAM, and importance of Color and Pulsed Doppler sonography in detection pseudoaneurysm within the neoplasm. Because of the nonspecific symptoms and high rate of complications, the detection of such a vascular lesion should prompt appropriate treatment based on the size and the risk of hemorrhage of RAMLs.

\section{Acknowledgments}

Funding: None.

\section{Footnote}

Peer Review File: Available at http://dx.doi.org/10.21037/ apm-20-475

Conflicts of Interest: All authors have completed the ICMJE uniform disclosure form (available at http://dx.doi. org/10.21037/apm-20-475). The authors have no conflicts of interest to declare.

Ethical Statement: The authors are accountable for all aspects of the work in ensuring that questions related to the accuracy or integrity of any part of the work are appropriately investigated and resolved. All procedures performed in studies involving human participants were in accordance with the ethical standards of the institutional and/or national research committee(s) and with the Helsinki Declaration (as revised in 2013). Written informed consent was obtained from the patient for publication of this manuscript and any accompanying images.

Open Access Statement: This is an Open Access article distributed in accordance with the Creative Commons Attribution-NonCommercial-NoDerivs 4.0 International License (CC BY-NC-ND 4.0), which permits the noncommercial replication and distribution of the article with the strict proviso that no changes or edits are made and the original work is properly cited (including links to both the formal publication through the relevant DOI and the license). See: https://creativecommons.org/licenses/by-nc-nd/4.0/.

\section{References}

1. Iruloh C, Keriakos R, Smith DJ, et al. Renal angiomyolipoma and lymphangioleiomyomatosis in pregnancy. J Obstet Gynaecol 2013;33:542-6.

2. Schieda N, Kielar AZ, Al Dandan O, et al. Ten uncommon and unusual variants of renal angiomyolipoma (AML): radiologic-pathologic correlation. Clin Radiol 2015;70:206-20.

3. Zapardiel I, Delafuente-Valero J, Bajo-Arenas JM. Renal angiomyolipoma during pregnancy: review of the literature. Gynecol Obstet Invest 2011;72:217-9.

4. Ferianec V, Gábor M, Caňo M, et al. Severe retroperitoneal haemorrhage in the first trimester of a multiple pregnancy after spontaneous rupture of renal angiomyolipoma. Arch Gynecol Obstet 2013;288:1193-4.

5. Randle SC, Tuberous Sclerosis Complex: A Review. Pediatr Ann 2017;46:e166-71.

6. Schieda N, Hodgdon T, El-Khodary M, et al. Unenhanced CT for the Diagnosis of Minimal-Fat Renal Angiomyolipoma. AJR Am J Roentgenol 2014;203:1236-41.

7. Sharma G, Jain A, Sharma P, et al. Giant exophytic renal angiomyolipoma masquerading as a retroperitoneal 
liposarcoma: A case report and review of literature. World J Clin Oncol 2018;9:162-6.

8. Albi G, del Campo L, Tagarro D. Wunderlich's syndrome: causes, diagnosis and radiological management. Clin Radiol 2002;57:840-5.

9. Sparks D, Chase D, Thomas D, et al. The Wunderlich's syndrome secondary to massive bilateral angiomyolipomas associated with advanced tuberous sclerosis. Saudi J Kidney Dis Transpl 2011;22:534-7.

10. Shankar S, Hulikanthimatt KS, Awatti SM, et al. Bilateral Multifocal Renal Angiomyolipoma Associated with Wunderlich's Syndrome in A Tuberous Sclerosis Patient. J Clin Diagn Res 2015;9:ED01-3.
Cite this article as: Chong J, Zhang J, Ning C, Zhang L, Zhao W, Sun Y. Fat-poor renal angiomyolipoma combined with pseudoaneurysm: a case report. Ann Palliat Med 2021;10(2):23432348. doi: 10.21037/apm-20-475 